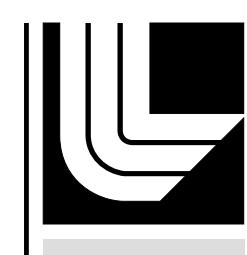

Design and simulations of indirect drive ignition targets for NIF

S. W. Haan, P. A. Amendt, T. R. Dittrich, B. A. Hammel, S. P. Hatchett, M. C. Herrmann, O. A. Hurricane, O. S. Jones, J. D. Lindl, M. M. Marinak, D. Munro, S. M. Pollaine, J. D. Salmonson, G. A. Strobel, L. J. Suter

November 2, 2004

Nuclear Fusion 
This document was prepared as an account of work sponsored by an agency of the United States Government. Neither the United States Government nor the University of California nor any of their employees, makes any warranty, express or implied, or assumes any legal liability or responsibility for the accuracy, completeness, or usefulness of any information, apparatus, product, or process disclosed, or represents that its use would not infringe privately owned rights. Reference herein to any specific commercial product, process, or service by trade name, trademark, manufacturer, or otherwise, does not necessarily constitute or imply its endorsement, recommendation, or favoring by the United States Government or the University of California. The views and opinions of authors expressed herein do not necessarily state or reflect those of the United States Government or the University of California, and shall not be used for advertising or product endorsement purposes. 


\title{
Design and simulations of indirect drive ignition targets for NIF
}

\author{
S. W. Haan, P. A. Amendt, T. R. Dittrich, B.A. Hammel, S. P. Hatchett, M. C. Herrmann, \\ O. A. Hurricane, O. S. Jones, J.D. Lindl, M. M. Marinak, D. Munro, S. M. Pollaine, \\ J. D. Salmonson, G. A. Strobel, ${ }^{1}$ and L. J. Suter \\ Lawrence Livermore National Laboratory, Livermore CA, 94551, haan1@1lnl.gov \\ ${ }^{1}$ also at Phys Department, Univ of Georgia, Athens GA \\ PACS 52.57.Bc
}

\begin{abstract}
Effort on simulation and design of ignition targets for the National Ignition Facility is described. Recent effort has emphasized systematic exploration of the parameter space of possible ignition targets, providing as specific as possible comparisons between the various targets. This is to provide guidance for target fabrication R\&D, and for the other elements of the ignition program. Targets are being considered that span 250-350 eV drive temperatures, capsule energies from 150 to $600 \mathrm{~kJ}$, cocktail and gold hohlraum spectra, and three ablator materials $(\mathrm{Be}[\mathrm{Cu}], \mathrm{CH}[\mathrm{Ge}]$, and polyimide). Capsules with graded doped beryllium ablators are found to be very stable with respect to short-wavelength Rayleigh-Taylor growth. Sensitivity to ablator roughness, ice roughness, and asymmetry is being explored, as it depends on ablator material, drive temperature, and absorbed energy. Three-dimensional simulations are being used to ensure adequate radiation symmetry in 3D, and to ensure that coupling of 3D asymmetry and 3D Rayleigh-Taylor does not adversely affect planned performance. Integrated 3D hohlraum simulations indicate that 3D features in the laser illumination pattern affect the hohlraums' performance, and the hohlraum has been redesigned to accommodate these effects.
\end{abstract}

\section{INTRODUCTION}

This article is an update on the simulation and design effort at LLNL, for targets meant to achieve ignition using indirect drive on the National Ignition Facility [1]. Targets were originally designed as described in refs. [2] and [3]. More recent updates on the developing designs, and the physics basis for them, are in refs. [4-10]. A baseline design is shown in Fig. 1. Detailed simulations are done with the codes LASNEX [11] in one and two dimensions (2D), and HYDRA [12,13] in one to three dimensions (3D). Targets are being considered that span 250-350 eV drive temperatures [10,14], capsule energies from 150 to $600 \mathrm{~kJ}$, cocktail and gold hohlraum spectra, and three ablator materials ( $\mathrm{Be}[\mathrm{Cu}], \mathrm{CH}[\mathrm{Ge}]$, and polyimide). Describing all of these is beyond the scope of this paper; representative results are described below. 
We are currently emphasizing two areas. First, we are exploring options for making the target designs more robust. This effort includes better capsule optimization, graded $\mathrm{Cu}$ dopant in $\mathrm{Be}$, and hohlraum design modifications. These are all described in more detail below. Secondly, we continue to pursue more detailed modeling of select point designs in order to enhance our confidence in their performance. Recently this category of effort has concentrated on 3D hohlraum design, culminating in a new design with optimized symmetry in 3D and an implosion simulation that includes this asymmetry plus fabrication defects in all modes. Details of all this work are presented in the following. Recent updates on target fabrication specifications are in refs. [15-16].

The basic design concept, shown in Fig. 1, remains the same as it has been since NIF was proposed: a cylindrical hohlraum about $1 \mathrm{~cm}$ long, filled with $\mathrm{He}$ or $\mathrm{H}+\mathrm{He}$ gas enclosed in $1 \mu \mathrm{m} \mathrm{CH}$ or polyimide windows, and illuminated with $0.3 \mu \mathrm{m}$ light. The capsule is a plastic or beryllium single shell ablator, supported by a tent, enclosing a cryogenic solid DT layer. While the basic design remains the same, many details are being optimized as explained below.

First, in the area of hohlraum design, we have been working in several areas. The hohlraum is being re-optimized in $3 \mathrm{D}$ as described in more detail below. Also, designs using $0.5 \mu \mathrm{m}$ light may allow for considerably more energy delivered to the capsule, albeit possibly at lower drive temperature. That work is described in more detail in the article by Suter in this issue. The efficiency of the hohlraum may be extended considerably from the original assumptions $[2,3]$ by careful optimization [14]. From the point of view of capsule design and optimization, the color of the light and the efficiency of the hohlraum only affect where one is likely to operate in the parameter space of capsule size and drive temperature. Of course higher absorbed energy is very valuable to capsule performance; one example that has been examined in some detail is uniformly doped $\mathrm{Be}(\mathrm{Cu})$ capsules at $250 \mathrm{eV}$, for which the surface roughness specification increases from $10 \mathrm{~nm}$ (the so-called "NIF standard" surface roughness [15]) at $250 \mathrm{~kJ}$ absorbed, to $60 \mathrm{~nm}$ at $600 \mathrm{~kJ}$ absorbed. Finally, the hohlraum designers are considering variants on the lining and fill of the hohlraum, which are also described in Suter's paper.

A qualitatively different design, that is not described here, is a double shell [17]. This design has the advantages that no cryogenics is required, and the pulse-shaping is much simpler. It has the disadvantage that there is more Rayleigh-Taylor growth at the interfaces in the capsule, and that this growth is very difficult to model.

\section{CAPSULE OPTIMIZATION}

We continue to refine the capsule design with simulations and experiments. The hohlraum design and laser performance will eventually give us constraints on capsule size, pulse shape, and drive temperature. The main question we need to answer now, to guide target fabrication and the other elements of the program, is how do we optimize the capsule performance given these constraints?

A new way to provide this optimization has been developed by one of us (Mark Herrmann). Herrmann's innovations have been a better implementation of the constraints than previously, and an automated process that thoroughly optimizes over the remaining free parameters. Ultimately the true constraints are on hohlraum size, as that determines the hohlraum energetics, and the temperature vs. time profile as determined by the laser power and energy. These in turn constrain the capsule size, as that affects case-to-capsule ratio, the peak temperature $T_{R}$, and, to a first 
approximation, the time integral of $\mathrm{T}_{\mathrm{R}}{ }^{4}(\mathrm{t})$. This is different from past work, where we considered the absorbed energy as being the primary quantity to hold fixed. Herrmann argues that capsules with lower albedos, that can absorb more energy at given size and $T_{R}$, should not be "punished" for this better performance. Thus the capsule absorbed energy is not constrained in the optimization, although of course it does not vary very much given the constraint on outer radius, peak $\mathrm{T}_{\mathrm{R}}$, and integral of $\mathrm{T}_{\mathrm{R}}{ }^{4}(\mathrm{t})$.

Given that we fix these three parameters, a capsule design is still characterized by ablator material, ablator thickness, fuel thickness, and the detailed shape of $\mathrm{T}_{\mathrm{R}}(\mathrm{t})$. For a given ablator material, and assuming that we can optimize $\mathrm{T}_{\mathrm{R}}(\mathrm{t})$, that leaves a two-dimensional parameter space of fuel thickness and ablator thickness. At each point in this space, the drive profile is automatically adjusted by a set of scripts running LASNEX and adjusting the profile to produce the shock timing determined to optimal by Munro [19]. This scan over dimensions then provides a map of performance in the two dimensional parameter space of shell thicknesses, as shown in Fig. 2. When the ablator is too thin, it burns through and the target fails to ignite. When the shells are too thick, the velocity is inadequate and again the target fails. In between is the operating space.

Having defined the space of one-dimensional performance, we can then explore the 2D (and presumably 3D)

characteristics. As one might expect, we find that short-wavelength Rayleigh-Taylor is generally worse as we move towards thinner shells within this space, while sensitivities to longer wavelength target fab defects, or to asymmetry, depend more on deceleration Rayleigh-Taylor and accordingly are worse for the lower velocity targets with thicker shells. Because the short-wavelength Rayleigh-Taylor has more e-foldings of growth, it is generally more sensitive, i.e. the overall optimization tends to be more influenced by the short wavelengths than the long, pushing us towards thicker shells than previously thought optimal. Once the implosion velocity is above about $37 \mathrm{~cm} / \mu \mathrm{s}$ (at $1.11 \mathrm{~mm}$ outer radius for plastic capsules) the long wavelength robustness does not increase very much with velocity, so the optimum design is probably that which has velocity just at $37 \mathrm{~cm} / \mu \mathrm{s}$. While this specific optimization is valuable, the most important product of this work is a quantification of the tradeoff, and the creation of the tools to evaluate the whole space of targets. Future work will change the specifics, and tell us which source of perturbation is dominant; now we have the tools to evaluate the relative importance of future results.

This scan technique has been applied to a variety of targets, including: the polyimide "scale 1" $300 \mathrm{eV}$ capsule shown in Fig. 2; $\mathrm{CH}(\mathrm{Ge})$ at the same outer radius and peak drive; and three ablator materials at $250 \mathrm{eV}$ : $\mathrm{CH}$ (undoped), polyimide, and $\mathrm{Be}(\mathrm{Cu})$. We find that at $250 \mathrm{eV}$, undoped $\mathrm{CH}$ has similar yield to uniformly doped $\mathrm{Be}(\mathrm{Cu})$, and can tolerate similar surface roughness (about $75 \mathrm{~nm}$ in spherical harmonic modes above 10, assuming a standard surface power spectrum, at a scale where the capsule absorbs $350 \mathrm{~kJ}$ ). At this low drive temperature, both are considerably superior to polyimide, which gives similar yield but requires the ablator surface to be a factor of several smoother.

\section{GRADED DOPED BERYLLIUM}

In addition to the optimization over capsule dimensions, we have examined the advantages of radially grading the $\mathrm{Cu}$ dopant in beryllium capsules. We find a surprising improvement in performance. 
Graded dopants have been considered previously, in fact the original NIF designs used graded $\mathrm{NaBr}$ as dopants in $\mathrm{Be}$, as was the $250 \mathrm{eV}$ design describe in Lindl [3]. These very early designs were not optimized in any detail, and used monotonically decreasing dopant concentration. Somewhat more recently, Dittrich [18] did a detailed optimization of a graded doped capsule meant to be driven at very low energy and temperature (115 kJ absorbed and $250 \mathrm{eV}$ respectively). Dittrich used a grading scheme proposed by Hatchett (unpublished). At that scale, Dittrich's design was not very robust, but as further work was done we continued to find that other $250 \mathrm{eV}$ targets were noticeably less robust than Dittrich's design. Hence we reexamined this design, examining its performance at larger more typical NIF scales, as well as considering a similar $300 \mathrm{eV}$ design.

These designs are much less susceptible to acceleration-phase Rayleigh-Taylor growth than any other ignition target that we have modeled. Fig. 3 shows the dimensions of the $300 \mathrm{eV}$ design, and summarizes the Rayleigh-Taylor results for the cases we have examined. The quantity tabulated as "ablator cliff" is the ablator surface roughness, with the NIF standard spectrum, which causes yield degradation to 50\% of that obtained in unperturbed 1D simulations.

The remarkable stability of these designs is evidently a result of the density gradient that the graded dopant creates in the ablating beryllium. Fig. 4 shows density profiles from Hydra simulations of three cases: a typical uniformly doped $\mathrm{Be}(\mathrm{Cu})$ capsule, the graded doped capsule, and a hypothetical undoped beryllium ablator. All are shown at a radius corresponding to about $2 / 3$ of the initial radius. The implosion with the undoped ablator is clearly unstable at the interface between the ablator and the fuel. This implosion would have very serious short-wavelength Rayleigh-Taylor growth. The uniformly doped ablator reduces the short wavelength growth by providing a better match to the fuel density, but at the price of substantial instability at the ablation front. In designing a uniformly doped capsule, one must choose the dopant concentration to trade off these two instabilities optimally. The graded dopant allows for both a minimal instability at the ablation front, which is undoped Be for much of the implosion, and little or no instability at the fuel/ablator interface, where the dopant gradient brings the ablator density smoothly into agreement with the fuel density. A key feature of this scheme, missing from most past graded-doped NIF capsules, is the presence of undoped Be adjacent to the fuel. This material couples less to preheat than the doped material, hence staying cooler and at higher density. The mass ablation rate is also higher, since much of the ablator is low-opacity undoped Be, and the doped layers can have lower dopant concentration than is necessary in a uniformly doped ablator.

Even though sputtered beryllium surfaces have generally been considered unacceptably rough, these designs are so stable that they can tolerate existing sputtered surface roughness. The technology of constructing graded layers is probably a straightforward extension of sputtered beryllium fabrication technology: one simply changes, as a function of time, the copper concentration in the thickening layer. The issues remain of filling and characterizing the layer, as in all beryllium capsules, but the additional stability of these capsules probably make them more forgiving of the possible solutions to these issues.

The $300 \mathrm{eV}$ capsule is also very tolerant of ice perturbations. The thick ablator and high ablation velocity make for low feed-out and low growth. The capsule can tolerate up to $5 \mu \mathrm{m} \mathrm{rms} \mathrm{ice} \mathrm{perturbations,} \mathrm{using} \mathrm{the} \mathrm{old} \mathrm{spectrum} \mathrm{as}$ described in ref. 2. (There is a typo in the ice spectrum equation in ref. 2, the numerical coefficient should be $2.2 \times 10^{-7}$ ) The $5 \mu \mathrm{m} \mathrm{rms} \mathrm{ice} \mathrm{roughness} \mathrm{produces} \mathrm{yield} \mathrm{of} 50 \%$ of clean. For this spectrum with $5 \mu \mathrm{m}$ rms total, the rms in spherical 
harmonic modes 10-15, which are likely to be the most important, is $1.4 \mu \mathrm{m}$. For comparison, typical recent Omega ice surfaces [20] can be described as approximately

$$
\mathrm{R}_{\mathrm{lm}}=10 \mu \mathrm{m} l^{-1.8}
$$

Such a spectrum has total $\mathrm{rms} 5.5 \mu \mathrm{m}$, which is somewhat higher than the best Omega surfaces of $1.8 \mu \mathrm{m}$ rms. With this $l^{-1.8}$ spectrum, with a total rms of $5.5 \mu \mathrm{m}$, the rms in the important modes $10-15$ is $0.39 \mu \mathrm{m}$. Hence we can tentatively draw the remarkable conclusion that this capsule could tolerate "typical" Omega ice roughness, with a safety factor of more than 4. This conclusion does not extend to modes below 10, for which (see below) we expect the specification to be similar to the current typical requirement. Then the spectrum given above is a factor of about 5 larger than the specification.

We have also examined the sensitivity of the $300 \mathrm{eV}$ graded-dopant capsule to low mode asphericity. Its sensitivity is very similar to the baseline polyimide capsule - both the growth factors for low modes, and the amount of bangtime low mode perturbation that the capsule can tolerate, are virtually identical to the baseline. The extraordinary features of this capsule are only evident for perturbations with spherical harmonic mode number greater than about 20.

Because these capsules are so robust with respect to short wavelengths, there may be a better optimization with somewhat thinner shells producing a higher velocity implosion that is more tolerant of low mode irregularities while still having satisfactory behavior with respect to high modes. That is an obvious area of future work. Another important area of future work for these capsules is examining how large of a fill tube they can tolerate, since that is a likely fill scenario, and the short wavelength stability may make for a relatively loose specification.

\section{3D SIMULATIONS}

Our program of 3D simulations with the code HYDRA [12,13] continues to advance. For several years we have been doing simulations of the capsule alone; Hydra now has all of the physics necessary to do 3D integrated simulations of the hohlraum and the capsule together, with IMC radiation transport and non-LTE modeling of the gold wall.

Three dimensional simulations are fast enough to be practical, although the biggest calculations we do require massively parallel computers. An integrated hohlraum+capsule simulation, with 15 million IMC photons, 1200 rays/beam laser raytrace, nonLTE from XSN, and Arbitrary Lagrange-Eulerian grid motion, takes a few days on a multi-processor machine. Capsule only implosions with either asymmetry alone (with more solid angle but less angular resolution), or with Rayleigh-Taylor alone (with less solid angle but more angular resolution) are similar. The biggest calculations are capsule implosions with both asymmetry and Rayleigh-Taylor; the one described below has 177 radial, 161 azimuthal, and 451 polar zones. With multi-group radiation diffusion, charged particle transport, and neutron freestreaming plus energy deposition, such a simulation takes a couple of weeks on a significant fraction of a massively parallel ASCI computer.

The 3D simulations of the NIF hohlraum suggested a number of design improvements. The pattern of energy flow through the LEH is three dimensional, and the LEH blows up somewhat more in $3 \mathrm{D}$ than in $2 \mathrm{D}$ because of the 
proximity of high intensity beams to the LEH lip at some locations. This was adjusted by increasing the thickness of the $\mathrm{CH}$ liner on the LEH from 20 to $30 \mu \mathrm{m}$. (The original nominal value was in fact $30 \mu \mathrm{m}$, but a tightening of the optimization in $2 \mathrm{D}$ had led to a reduction to $20 \mu \mathrm{m}$.) Also, the lowest azimuthal periodicity of the NIF beam spots is $\mathrm{m}=4$ - the innermost cones at $23.5^{\circ}$ and $30^{\circ}$ have 4 beams each, which caused a $\mathrm{Y}_{54}$ asymmetry on the capsule with the original pointing. This was improved by repointing these eight beams to the same Z-coordinate on the hohlraum wall, so that the spot pattern early in time is closer to an 8-fold pattern.

This redesign was done with a cocktail-wall hohlraum $10.5 \mathrm{~mm}$ long. The capsule was a polyimide ablator, radii 946, 1034, and $1193.5 \mu \mathrm{m}$. It is driven with $1.49 \mathrm{MJ}$ of $0.3 \mu \mathrm{m}$ light, peak power $390 \mathrm{TW}$, in an $18.5 \mathrm{~ns}$ pulse.

The result of these improvements in the hohlraum design, and then reoptimizing the cone-to-cone beam balance, was a hohlraum design with very good symmetry. The imploded shape at ignition time is shown in Fig. 5. A slight skewing is visible from the remaining $(5,4)$ asymmetry, and there are small spikes on the hohlraum axis (the horizontal axis in Fig. 5) This remaining asymmetry is typical of well-tuned integrated calculations and is completely acceptable. The rms deformation of the hot-spot at ignition time is $3 \mu \mathrm{m}$, which a factor of two or more smaller than what is needed to quench ignition.

Having achieved this asymmetry, we imposed it on a series of capsule-only implosions. This is done firstly with the goal merely to determine what time-dependent asymmetry faithfully reproduces the implosion in the integrated simulation (the asymmetry used for the capsule-only implosions is inferred from the integrated calculation by analyzing the ablation pressure in the latter, as a function of angle, and using a non-Planckian flux source in the former which produces the same pressure moments). Having reproduced the asymmetry, we reduce the solid angle to a size more feasible for Rayleigh-Taylor calculations. This is necessary because the full 3D asymmetry in the NIF geometry requires $4 \pi$ solid angle. By combining amplitudes in some spherical harmonic modes, all the salient features can be reproduced in an implosion that is $90^{\circ}$ in $\theta$ and $45^{\circ}$ in $\phi$. In this geometry, high mode Rayleigh-Taylor can be included using the zoning described above.

A simulation that has all of the nominal deviations from sphericity, including the 3D hohlraum asymmetry, gives $21 \mathrm{MJ}$ of yield. This included a "NIF standard" spectrum of perturbations on the ablator, including the low modes for which the NIF standard is very similar to the published specification. [16] Low mode ice perturbations were set to the published specification, and high modes were given a power-law shape typical of recent Omega cryo target characterization (as described above) with the amplitudes scaled so that the rms was equal to $1.7 \mu \mathrm{m}$ which they have seen on their best surfaces. The simulation was done over the $90^{\circ}$ by $45^{\circ}$ wedge described above. The surface perturbations had randomly selected $\operatorname{Re}\left(Y_{l m}\right)$ modes, with specified power as a function of the mode number $l$ alone. Only even $l$ are included, and $m$-values that are multiples of 4 , with power that would be in other modes included in the allowed modes. This makes an isotropic perturbation compatible with reflecting boundary conditions, with the desired power spectrum.

Fig. 6 shows the configuration at peak velocity, and Fig. 7 at ignition time. The overall shape is very similar to Fig. 5 (note the figures are rotated $90^{\circ}$ with respect to each other), but the short-wavelength modes are evident. The amplitudes of these modes are not sufficient to quench the ignition, and the target burns robustly. 


\section{SUMMARY}

This article has described several advances in our modeling and understanding. Systematic scans of the parameter space of possible capsules have resulted in significant improvements in simulated performance, providing more margin for looser specifications or errors in our understanding. We have found that radially grading the dopant concentration in beryllium capsules produces a remarkable increase in the short-wavelength stability. And finally, simulations in 3D have indicated hohlraum design improvements, and demonstrated good performance in simulations that include all nominal sources of deviation. Overall, these results continue to increase our confidence in achieving ignition with indirect drive on NIF. 


\section{ACKNOWLEDGEMENT}

This work was performed under the auspices of the U.S. Department of Energy by the University of California, Lawrence Livermore National Laboratory under contract No. W-7405-Eng-48.

\section{REFERENCES}

[1] Campbell E.M. and Hogan W.J. 1999 Plasma Phys. Control. Fusion 41 B39-B56

[2] Haan S.W. et al. 1995 Phys. Plasmas 22480

[3] Lindl J.D. 1998 Inertial Confinement Fusion, Springer-Verlag, New York (1998), p. 162

[4] Krauser W.J. et al. 1996 Phys. Plasmas 32084

[5] Wilson D.C. et al. 1998 Phys. Plasmas 51953

[6] Bradley P.A. and Wilson D.C. 1999 Phys. Plasmas 64293

[7] Bradley P.A. and Wilson D.C. 2001 Phys. Plasmas 83724

[8] Dittrich T.R. et al. 1999 Phys. Plasmas 62164

[9] Lindl J.D. et al. 2004 Phys. Plasmas 11339

[10] Hinkel D.E. et al. 2004 Phys. Plasmas 111128

[11] Zimmerman G. and Kruer W.L. 1975 Comments Plasma Phys. Controlled Fusion 285

[12] Marinak M.M. et al. 1998 Phys. Plasmas 51125

[13] Marinak M.M. et al. 2001 Phys. Plasmas 82275

[14] Suter L. et al. 2000 Phys. Plasmas 72092

[15] Haan S.W. et al. 2002 Fusion Science and Tech. 41165

[16] Strobel G.L. et al. 2004 Phys. Plasmas 11, 1617

[17] Amendt P.A. et al. 2002 Phys. Plasmas 92221

[18] Dittrich T.R. et al. 1998 Phys. Plasmas 53708

[19] Munro D.H. et al. 2001 Phys. Plasmas 82245

[20] Warrick A.L. 2003 A shadowgraphic analysis procedure for cryogenic layer characterization Target Fabrication Specialists Meeting, Gleneden Beach, Oregon June 2003 
Figure captions

Figure 1. Baseline NIF ignition target. The hohlraum, upper left, is about $1 \mathrm{~cm}$ long and $5.5 \mathrm{~mm}$ in diameter. It is made of either gold or a mixture with optimized opacity, called a cocktail. The central spherical capsule is shown blown up in the pie diagram, lower left. The laser power, and corresponding radiation temperature in the hohlraum, is shown on the right. This baseline target uses $1.3 \mathrm{MJ}$ of $0.3 \mu \mathrm{m}$ light, at peak power $410 \mathrm{TW}$. The capsule absorbs $150 \mathrm{~kJ}$, and produces $15 \mathrm{MJ}$ of fusion energy.

Figure 2. Yield of polyimide capsules vs. shell thicknesses. At each set of dimensions the pulse-shape driving the capsule is optimized by an automated routine. The outer radius is held fixed at $1.110 \mathrm{~mm}$, the peak $\mathrm{T}_{\mathrm{R}}$ at $300 \mathrm{eV}$, and the integral of $\mathrm{T}_{\mathrm{R}}{ }^{4}(\mathrm{t})$ is held fixed at a scale corresponding to $1.3 \mathrm{MJ}$ of laser energy. Two velocity contours are indicated, in $\mathrm{cm} / \mathrm{s}$.

Figure 3. Be capsule with graded $\mathrm{Cu}$ dopant. The table shows results on 4 designs; the $300 \mathrm{eV}$ design is shown, while the $250 \mathrm{eV}$ designs are scales of the design by Dittrich (ref. 17). The table shows how large of an ablator perturbation causes $50 \%$ yield degradation.

Figure 4. Density profiles in imploding capsule for three dopants in beryllium ablators, shown at about 2/3 initial radius. Thickened lines show regions where the density profile is most unstable.

Figure 5. Density isocontours at ignition time for a 3D integrated simulation. The density scale ranges linearly from 0 to $1386 \mathrm{~g} / \mathrm{cc}$, and the frame is $110 \mu \mathrm{m}$ square.

Figure 6. 3D implosion with full asymmetry and nominal fabrication defects in low and high modes. This shows the $60 \mathrm{~g} / \mathrm{cc}$ isosurface $140 \mathrm{ps}$ before ignition time. Hohlraum axis is vertical. On the right side, with the contours hidden in the first quadrant, we see the inner $60 \mathrm{~g} / \mathrm{cc}$ surface showing the perimeter of the hot-spot. On the left side the contour picks out the DT/polyimide interface with short wavelength perturbations.

Figure 7. Same 3D implosion as Fig. 6, at ignition time, i.e. when the central $T_{i}$ is $10 \mathrm{keV}$. The scale is enlarged relative to fig. 6. Hohlraum axis is vertical. This object is the same size as Fig. 5 (the $400 \mathrm{~g} / \mathrm{cc}$ contour). On the right cutaway side, the $400 \mathrm{~g} / \mathrm{cc}$ surface shows the perimeter of the hot-spot, while on the left side it shows the outgoing stagnation shock. As is also evident in Figs. 5 and 6, this target has a slightly prolate imploded shape. 

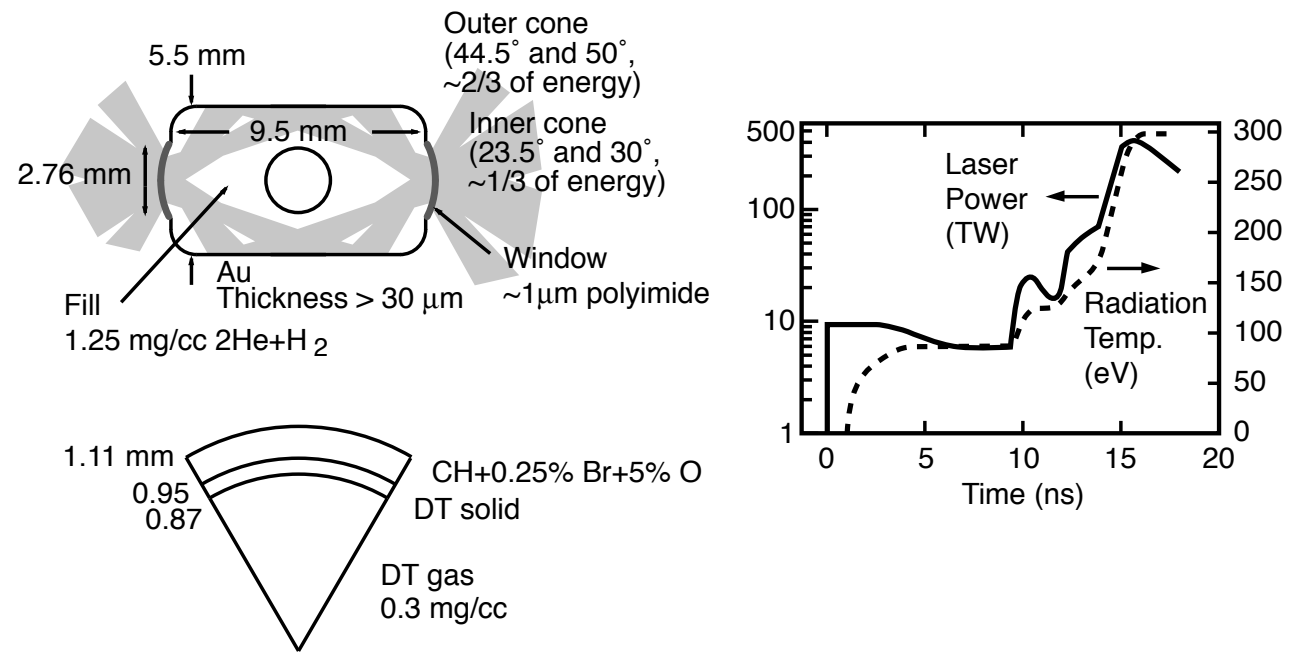

Capsule yield $15 \mathrm{MJ}$

Haan Fig. 1 


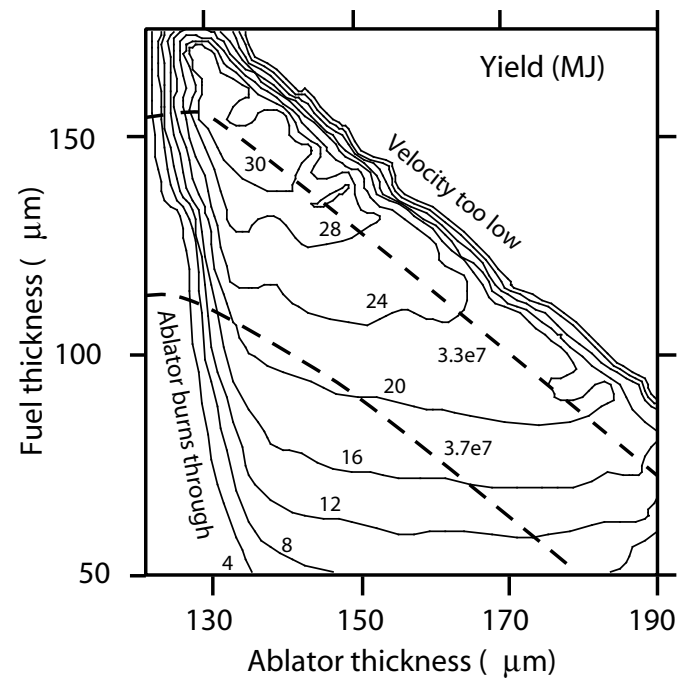

Haan Fig. 2 


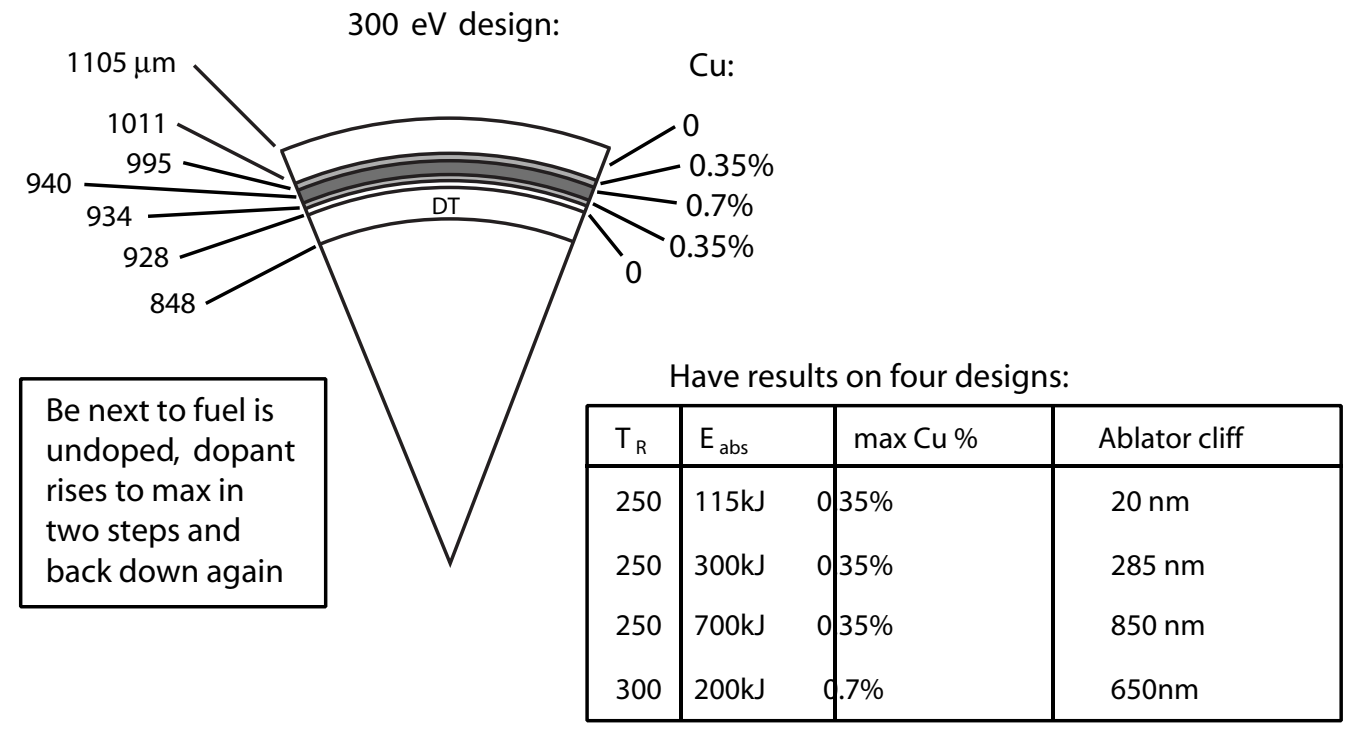

Haan Fig. 3 


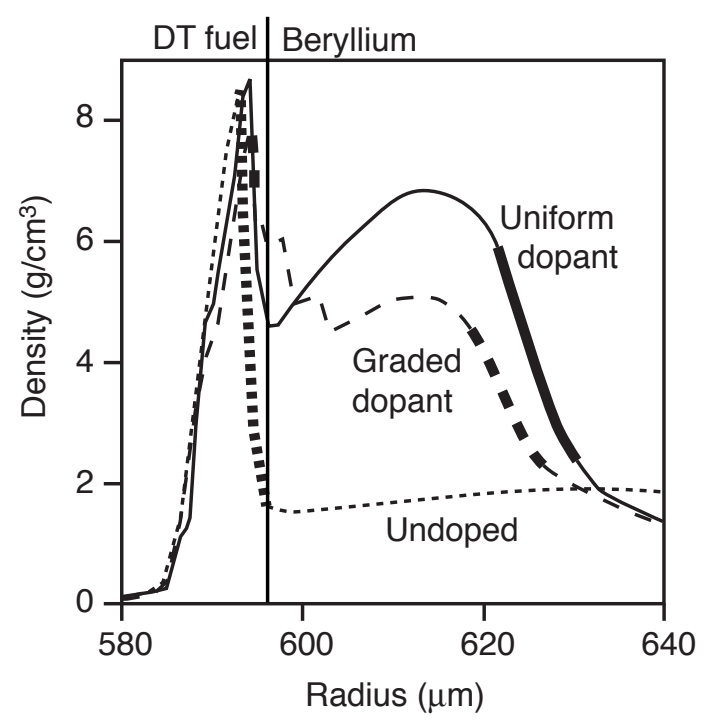

Haan Fig. 4 


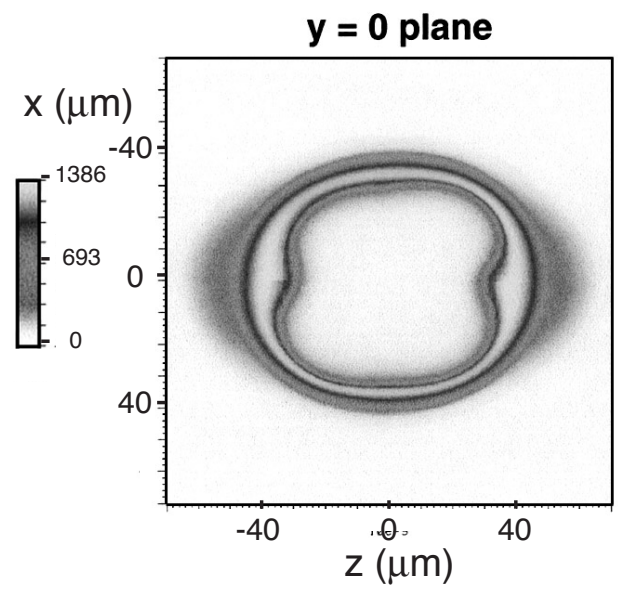

Haan Fig. 5 


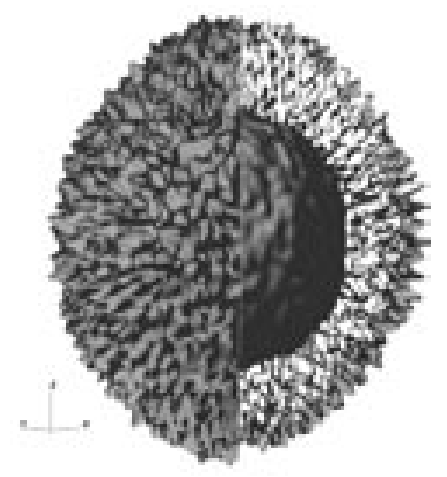




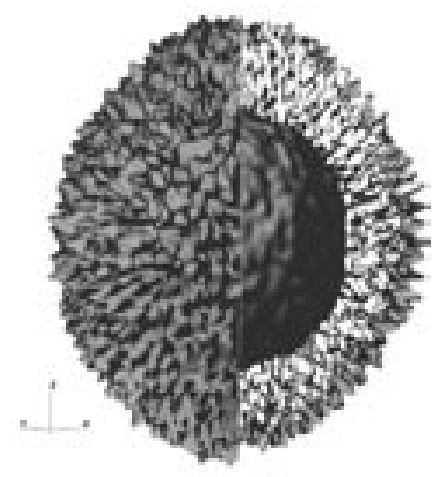




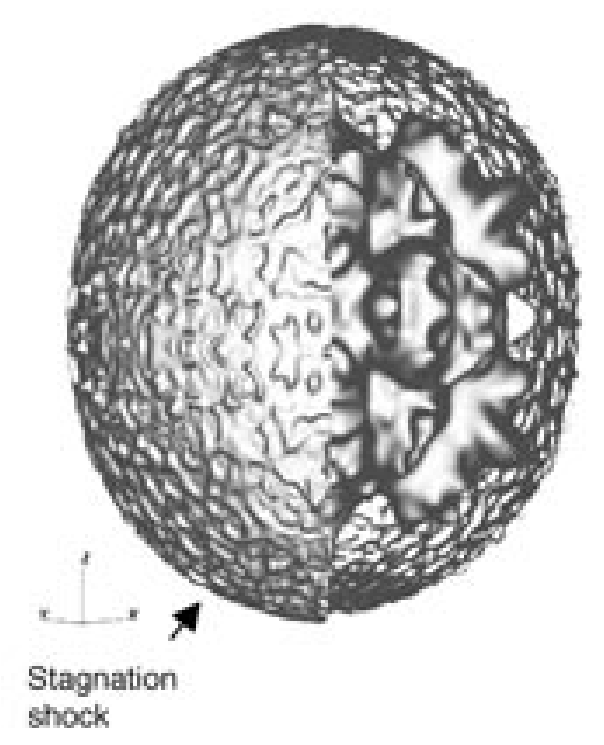

